\title{
Can Evidence-Based Medicine and Personalized Medicine Coexist?
}

\author{
Kim Madden and Mohit Bhandari
}

\subsection{What Is Evidence-Based Medicine?}

Evidence-based medicine (EBM) is a philosophy of healthcare that aims to ensure that healthcare interventions are applied based on the best available evidence, combined with clinical expertise and patient values [1]. This is in contrast to the philosophy of "eminence-based" medicine, which is characterized by a paternalistic view that expert clinicians know what is best for their patients by virtue of their clinical experience. The term EBM was coined by Professor Gordon Guyatt in 1990 and further developed by academic physicians such as Professor David Sackett. Sackett described EBM as having three integrated key components: best available evidence, clinical expertise, and patient values [1]. Here, we discuss each of these three components in more detail.

\subsubsection{Best Available Evidence}

It makes intuitive sense that healthcare professionals should be reasonably sure that a treatment works and that the benefits outweigh the

K. Madden $\cdot$ M. Bhandari $(\bowtie)$

Department of Surgery, McMaster University,

Hamilton, ON, Canada

e-mail:maddenk@mcmaster.ca;

bhandam@mcmaster.ca harms before wide implementation of the intervention. It is important to question unsubstantiated claims about treatments, diagnostic tools, and other aspects of healthcare so that we do not widely use treatments that are ineffective or do more harm than good. Using systematic and scientific methodology, EBM gives us the tools to evaluate healthcare interventions and determine how strong and convincing the evidence is for those interventions, and therefore whether we should believe claims of their efficacy. The phrase "best available evidence" implies that some evidence is better than other evidence. This brings us to one of the key principles of EBM: the hierarchy of evidence. Many healthcare professionals are aware of the "evidence pyramid" that places high-quality evidence on the top of the pyramid and low-quality evidence on the bottom of the pyramid [2]. EBM helps us to sort out which studies are high quality and which studies are low quality. However, this categorization is not binary; quality of evidence is a continuum. In general, the highest quality of evidence for questions about treatment efficacy comes from randomized controlled trials (RCTs) and systematic reviews of RCTs. The reason for this is that, when done correctly, the process of randomization should balance the known and unknown prognostic factors across treatment groups, with the only difference between groups being the treatment of interest. RCTs are not always at the top of the hierarchy of evidence. EBM also 
encourages downgrading evidence in the presence of substantial methodological flaws [3]. For example, if a study is too small to properly balance prognostic factors across groups, that could lead to the study being downgraded from the top level of evidence. Prospective cohort studies are often at the second tier of evidence (Level II evidence) because they lack the randomization process that aims to balance prognostic factors. They are therefore more biased and of lower quality. Retrospective studies are Level III evidence because they are subject to even more bias than prospective studies, for example, recall bias. Case series are Level IV evidence because they lack a control group. We therefore cannot be sure whether apparent treatment effects can actually be attributed to the treatment or some other effect such as time. Expert opinion is Level V evidence because opinions can easily be biased by personal views, conflicts of interest, and other factors such as confirmation bias. By applying a critical lens to studies, we can practice "enlightened scepticism" to be reasonably sure that the treatments that we choose to use are effective.

\subsubsection{Clinical Expertise}

Critics of EBM often protest that EBM downplays the role of the clinician's expertise in favor of a cold, calculating style of medicine based only on evidence [4]. This is not the case. Evidence is not a substitute for clinical training and experience. Evidence alone is never enough to make a clinical decision. The proper application of EBM requires the integration of expertise and evidence. The JAMA series on the Users Guides to the Medical Literature, a key EBM resource, gives guidance on how to evaluate whether particular evidence is applicable to specific patients [5]. It teaches clinicians to ask "Were the study patients similar to the patient in my practice?" To answer this question, clinicians must use their diagnostic expertise and judgment. For example, surgeons may decide that a study that included mostly elderly female patients with comorbidities would not necessarily apply to an elite male athlete, even if the evidence is of very high quality.

\subsubsection{Patient Values}

The third major component of EBM is the integration of patient values $[6,7]$. Although this point is the most often forgotten, it has been written into formal definitions of EBM since the 1990s [1]. Along with the best available evidence and the clinician's expertise, we must take into account the patient's preferences. For example, an active, newly retired man with hip osteoarthritis may place more value on implant longevity than a very elderly man. Similarly, a young tradeswoman with moderate knee arthritis may value whichever treatment option can get her back to work faster. This principle particularly emphasizes that EBM is not a set of rigid rules, nor is it a one-size-fitsall approach to treating patients.

\subsection{Are There any Drawbacks to EBM?}

EBM is not perfect and is ever-evolving. A major practical challenge is that performing EBM properly requires a lot of practice and skill. However, this is the same for any other skill, for example, arthroplasty surgeons train for a decade or more to become experts at joint replacement. Sometimes, feasibility issues arise in EBM, for example, to get the highest quality evidence (i.e., RCTs), it can take years and cost millions of dollars to do it correctly. However, there are quicker and cheaper designs that can be done if an RCT is not feasible. For example, one could conduct retrospective chart reviews with matched controls or statistical adjustments based on propensity scores. This design is not as strong as an RCT but can efficiently provide better evidence than anecdote alone. One of the biggest challenges of EBM is that sometimes policymakers and clinicians forget that evidence alone is not sufficient, and they create overly strict policies that they say are evidence based. There needs to be integration of clinical judgment and patient values, which is in harmony with the principles of personalized medicine. Another perceived drawback of EBM is the misconception that results from trials can never apply to individual patients; they only 
apply to the "average patient." However, EBM books [5] and workshops [8] give explicit guidance on how to apply EBM to individual patients.

\subsection{What Is Personalized Medicine?}

Personalized medicine is a philosophy of treatment that arose from genomics, with particular applications in cancer treatment. The idea is that patients can be stratified into risk groups (e.g., biomarker present vs. absent) and provided with personalized treatment based on that risk factor [9]. This philosophy has clear applications in orthopedics, particularly in arthroplasty where many patients are not satisfied with their replaced joint despite a lack of major complications [10]. Kinematic alignment techniques that restore individual joint anatomy and soft-tissue balance, custom implants that can more accurately mimic the natural joint, robotic surgery for more precise cuts, and $3 \mathrm{D}$ printing are all innovations that can benefit the field of orthopedics by individualizing particular aspects of patient care. This intuitively sounds like a good idea. However, custom implants and technological innovations can drive up costs of surgery. We need evidence that these interventions are worth the additional money.

\subsection{Are EBM and Personalized Medicine at Odds?}

When Professor Gordon Guyatt was asked this question, he responded with "we find this somewhat amusing" [11]. The idea that personalized medicine is the opposite of EBM, or that they are somehow at odds, stems from a fundamental misunderstanding of what EBM is and is not. Particularly, the misconceptions that EBM is dogmatic, do not take into account patient values or differences between patients, there is no room for clinical judgment, that only randomized trials matter, and that EBM is a static set of rules, are misconceptions that contribute to the divide between personalized medicine and EBM. Let us address these misconceptions.
- EBM is dogmatic. EBM is not dogma; it is a set of guidelines that helps us decide whether a healthcare intervention is effective and safe and whether the evidence applies to our patients. Individual expertise, decision making, and judgment come into play at every stage of EBM.

- EBM does not take into account patient values. One of the three basic principles of EBM is that patient values and differences between patients should be taken into account when choosing a treatment. There is a whole field dedicated to how this can best be achieved, for example, with the use of patient decision aids and shared decision making [12]. Additionally, EBM is beginning to involve patients as collaborators when designing research and selecting outcomes for studies [13].

- EBM does not take into account differences between patients. EBM gives guidance on subgroup analyses to take into account differences between patients [14] Subgroups allow us to draw different conclusions for different groups of patients by categorizing them by a prognostic variable of interest, just like "stratified medicine." For example, in the SRINT trial investigating reamed versus unreamed intramedullary nailing for tibia fractures, the treatment effects varied for patients with open fractures versus closed fractures [15].

- There is no room for clinical judgment. One of the three basic principles of EBM is that clinical judgment cannot be replaced by evidence alone. Clinical expertise is still required to decide whether the evidence can be applied to a specific patient.

- Only randomized trials matter. EBM acknowledges that there are many ways to obtain evidence. The existence of the hierarchy of evidence proves this. Sometimes, patients cannot be randomized for ethical or feasibility reasons. In this case, EBM would say that an RCT is not the best available evidence. EBM has also always had an option for an N-of-1 trial, which is a trial where a single patient is their own control group [16]. This N-of-1 approach allows clinicians to determine whether a treatment works for that specific 
patient and provides better evidence than anecdote alone.

- EBM is a static set of rules. EBM is not a set of rules (see point 1), and EBM is constantly evolving. Some of the newer innovations in EBM include better methods of disseminating evidence (e.g., OrthoEvidence; myorthoevidence.com), extending EBM concepts to diagnostic and prognostic studies as well as interventions (e.g., the work of the Grading of Recommendations, Assessment, Development and Evaluation (GRADE) group) [17], methods of synthesizing information quickly (e.g., BMJ Rapid Recommendations; bmj.com/rapid-recommendations), and ever-evolving methods of analyzing data, particularly non-RCT data.

\subsection{So, Can EBM and Personalized Medicine Coexist?}

Not only can EBM and personalized medicine coexist, they should coexist. Personalized medicine-based interventions contribute to the growing number of innovations in orthopedics and other fields. However, these interventions still need to be evaluated for effectiveness, cost-effectiveness, and safety before they are widely adopted, just as standard approaches need to be evaluated with a critical lens. For example, one could randomize patients to receive conventional unicompartmental knee arthroplasty (UKA) versus custom UKA. Such a study would combine the best of both worlds and promote innovation in our field. There is no reason that the philosophies of EBM and personalized medicine cannot work together.

\section{References}

1. Sackett DL, Rosenberg WM, Gray JA, Haynes RB, Richardson WS. Evidence based medicine: what it is and what it isn't. BMJ. 1996;312(7023):71-2.

2. Panesar SS, Philippon MJ, Bhandari M. Principles of evidence-based medicine. Orthop Clin North Am. 2010;41(2):131-8.

3. Guyatt GH, Oxman AD, Kunz R, Vist GE, Falck-Ytter Y, Schünemann HJ, GRADE Working Group. What is "quality of evidence" and why is it important to clinicians? BMJ. 2008;336(7651):995-8.
4. Wilson K. Evidence-based medicine. The good the bad and the ugly. A clinician's perspective. J Eval Clin Pract. 2010;16(2):398-400.

5. Guyatt GH, Haynes RB, Jaeschke RZ, et al. Users' guides to the medical literature: XXV. Evidencebased medicine: principles for applying the Users' guides to patient care. Evidence-based medicine working group. JAMA. 2000;284:290-6.

6. Kelly MP, Heath I, Howick J, Greenhalgh T. The importance of values in evidence-based medicine. BMC Med Ethics. 2015;16(1):69.

7. Guyatt G, Montori V, Devereaux PJ, Schünemann $\mathrm{H}$, Bhandari M. Patients at the center: in our practice, and in our use of language. ACP J Club. 2004;140(1):A11-2.

8. McMaster Evidence-Based Clinical Practice Workshops. https://ebm.mcmaster.ca/.

9. Academy of Medical Sciences. Stratified, personalised or P4 medicine: a new direction for placing the patient at the centre of healthcare and health education (Technical report). 2015. https://acmedsci.ac.uk/ download?f=file \&i=32644.

10. Gunaratne R, Pratt DN, Banda J, Fick DP, Khan RJK, Robertson BW. Patient dissatisfaction following total knee arthroplasty: a systematic review of the literature. J Arthroplast. 2017;32(12):3854-60.

11. Guyatt G, Jaeschke R. Evolution of EBM. Part 1: EBM and personalized medicine. Are they different? 2018. https://empendium.com/mcmtextbook/interviews/perspective/197445, evolution-of-ebm-part1-ebm-and-personalized-medicine-are-they-different.

12. Montori VM, Breslin M, Maleska M, Weymiller AJ. Creating a conversation: insights from the development of a decision aid. PLoS Med. 2007;4(8):e233.

13. Sacristán JA, Aguarón A, Avendaño-Solá C, Garrido P, Carrión J, Gutiérrez A, Kroes R, Flores A. Patient involvement in clinical research: why, when, and how. Patient Prefer Adherence. 2016;10:631-40.

14. Sun X, Ioannidis JP, Agoritsas T, Alba AC, Guyatt G. How to use a subgroup analysis: users' guide to the medical literature. JAMA. 2014;311(4):405-11.

15. SPRINT Investigators, Sun X, Heels-Ansdell D, Walter SD, Guyatt G, Sprague S, Bhandari M, Sanders D, Schemitsch E, Tornetta P 3rd, Swiontkowski M. Is a subgroup claim believable? A user's guide to subgroup analyses in the surgical literature. J Bone Joint Surg Am. 2011;93(3):e8.

16. Guyatt G, Jaeschke R, McGinn TPART. 2B1: therapy and validity. N-of-1 randomized controlled trials. In: Guyatt G, Rennie D, Meade MO, Cook DJ, editors. Users' guides to the medical literature. New York: McGraw-Hill: American Medical Association; 2002. p. $275 \mathrm{e} 90$.

17. Iorio A, Spencer FA, Falavigna M, Alba C, Lang E, Burnand B, McGinn T, Hayden J, Williams K, Shea B, Wolff R, Kujpers T, Perel P, Vandvik PO, Glasziou P, Schunemann H, Guyatt G. Use of GRADE for assessment of evidence about prognosis: rating confidence in estimates of event rates in broad categories of patients. BMJ. 2015;350:h870. 
Open Access This chapter is licensed under the terms of the Creative Commons Attribution 4.0 International License (http://creativecommons.org/licenses/by/4.0/), which permits use, sharing, adaptation, distribution and reproduction in any medium or format, as long as you give appropriate credit to the original author(s) and the source, provide a link to the Creative Commons license and indicate if changes were made.

The images or other third party material in this chapter are included in the chapter's Creative Commons license, unless indicated otherwise in a credit line to the material. If material is not included in the chapter's Creative Commons license and your intended use is not permitted by statutory regulation or exceeds the permitted use, you will need to obtain permission directly from the copyright holder.

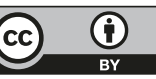

